\title{
HOLOMORPHE ÜBERLAGERUNGSKORRESPONDENZEN
}

\author{
HANS RISCHEL
}

0.

In der klassischen Abbildungstheorie im Raum von $n$ komplexen Veränderlichen $(n>1)$ wurden nur biholomorphe Abbildungen betrachtet. Es hat sich aber herausgestellt, dass auch das Studium allgemeinerer holomorpher Abbildungen von Bedeutung ist; u. a. spielen holomorphe Überlagerungsabbildungen in manchen neueren Arbeiten eine wichtige Rolle. Bei der Betrachtung holomorpher Überlagerungsabbildungen besteht die Schwierigkeit, dass keine Umkehrabbildung zur Verfügung steht. Diese Schwierigkeit verschwindet jedoch, wenn man auch mehrdeutige Abbildungen in Betracht zieht. In der vorliegenden Arbeit wird eine gewisse Klasse mehrdeutiger holomorpher Abbildungen (holomorphe Überlagerungskorrespondenzen genannt) untersucht. Als Verallgemeinerung eines Satzes von Osorio [3, Satz 6] wird bewiesen, dass holomorphe Überlagerungskorrespondenzen direkter Produkte von Gebieten mit speziellen Randeigenschaften in einem gewissen Sinne zerfallen (Satz 1). Als Anwendung dieses Satzes ergibt sich eine Verallgemeinerung der Poincaréschen Aussage über die Nichtabbildbarkeit des Dizylinders auf die Hyperkugel des $\boldsymbol{C}^{2}$. Weiter wird ein Satz von Remmert und Stein über holomorphe UUberlagerungsabbildungen analytischer Polyedergebiete [5, Satz 14] auf holomorphe Überlagerungskorrespondenzen ausgedehnt (Satz 2). Dieses Ergebnis ermöglicht eine Übersicht der holomorphen Überlagerungsabbildungen mit einem euklidischen Polyedergebiet genügend hoher Stufe als Urbild (Satz 4) oder Bild (Satz 5).

Es sei mir gestattet, an dieser Stelle Herrn Professor Dr. Karl Stein für die Anregung zu der vorliegenden Arbeit zu danken.

\section{1.}

Seien $X_{1}$ und $X_{2}$ reduzierte komplexe Räume [2, $\left.\S 1\right]$. Eine Korrespondenz $F: X_{1} \rightarrow_{k} X_{2}$ heisst eine holomorphe Überlagerungskorrespondenz, wenn $F$ und $F^{-1}$ holomorphe, surjektive, offene und nirgends ent-

Eingegangen am 13. März 1964.

Math. Scand. $15-4$ 
artete Korrespondenzen sind. Der Begriff der holomorphen Korrespondenz ist in $[9, \S 3.4]$ definiert. Eine Korrespondenz $F: X_{1} \rightarrow_{k} X_{2}$ heisst surjektiv, wenn jeder Punkt von $X_{2}$ Bildpunkt vermöge $F$ ist, offen, wenn jede offene Teilmenge von $X_{1}$ vermöge $F$ in eine offene Teilmenge von $X_{2}$ abgebildet wird, und nirgends entartet, wenn jede Menge der Gestalt $F^{-1}$ (Punkt) diskret ist.

Sind $X_{1}$ und $X_{2}$ komplexe Räume, so ist offenbar jede eigentliche holomorphe Úberlagerungsabbildung $f: X_{1} \rightarrow X_{2}([8, \S 1.1])$ eine holomorphe Überlagerungskorrespondenz.

Proposition 1. Es seien $X_{1}, X_{2}$ und $X_{3}$ komplexe Räume, $F_{1}, F_{2}$ : $X_{1} \rightarrow_{k} X_{2}$ und $G: X_{2} \rightarrow_{k} X_{3}$ holomorphe Überlagerungskorrespondenzen. Dann sind $F_{1}^{-1}: X_{2} \rightarrow_{k} X_{1}, G \circ F_{1}: X_{1} \rightarrow_{k} X_{3}$ und $F_{1} \cup F_{2}: X_{1} \rightarrow_{k} X_{2}$ ebenfalls holomorphe Überlagerungskorrespondenzen.

Die Korrespondenz $F_{1} \cup F_{2}$ ist durch

$$
\mathscr{G}\left[F_{1} \cup F_{2}\right]=\mathscr{G}\left[F_{1}\right] \cup \mathscr{G}\left[F_{2}\right]
$$

definiert. (Mit $\mathscr{G}[F]$ wird der Graph der Korrespondenz $F$ bezeichnet $[9, \S 3.1]$.)

In Proposition 1 ist nur nicht-trivial, dass $G \circ F_{1}$ eine holomorphe Überlagerungskorrespondenz ist. Nach einem Satz von Stein [9, Proposition 3.4.1] sind $G \circ F_{1}$ und $\left(G \circ F_{1}\right)^{-1}=F_{1}{ }^{-1} \circ G^{-1}$ holomorphe Korrespondenzen. Es ist klar, dass diese Korrespondenzen offen und surjektiv sind. Dass sie auch nirgends entartet sind, folgt sofort daraus, dass jede Menge $F^{-1}$ (Punkt) sogar endlich ist, wenn $F$ und $F^{-1}$ stetige und nirgends entartete Korrespondenzen sind.

Eine holomorphe Utberlagerungskorrespondenz $F: X_{1} \rightarrow_{k} X_{2}$ heisst irreduzibel, wenn der Graph $\mathscr{G}[F]$ eine in $X_{1} \times X_{2}$ irreduzible analytische Menge ist.

Proposition 2. Es seien $X_{1}$ und $X_{2}$ irreduzible komplexe Räume. Dann ist jede holomorphe Überlagerungskorrespondenz $F: X_{1} \rightarrow X_{2}$ Vereinigung von endlich vielen irreduziblen holomorphen Überlagerungskorrespondenzen $F_{i}: X_{1} \rightarrow_{k} X_{2}$.

BEWEIs. Es bezeichnen $\Pi_{1}: \mathscr{G}[F] \rightarrow X_{1}$ und $\Pi_{2}: \mathscr{G}[F] \rightarrow X_{2}$ die Restriktionen der natürlichen Projektionen $X_{1} \times X_{2} \rightarrow X_{1}$ und $X_{1} \times X_{2} \rightarrow$ $X_{2}$. Dann sind $\Pi_{1}$ und $\Pi_{2}$ offene Abbildungen. Sind nämlich $O_{1} \subseteq X_{1}$ und $O_{2} \cong X_{2}$ offene Mengen, so sind

und

$$
\Pi_{1}\left(\left(O_{1} \times O_{2}\right) \cap \mathscr{G}\left[F^{\prime}\right]\right)=O_{1} \cap F^{-1}\left(O_{2}\right)
$$

$$
\Pi_{2}\left(\left(O_{2} \times O_{2}\right) \cap \mathscr{G}[F]\right)=O_{2} \cap F\left(O_{1}\right)
$$


ebenfalls offen. Die Behauptung folgt dann aus der Definition der Topologie in $X_{1} \times X_{2}$.

Sei $M$ eine irreduzible Komponente des Graphen $\mathscr{G}[F]$. Sie enthält dann eine offene Teilmenge von $\mathscr{G}[F]$. Da $\Pi_{1}$ eine eigentliche holomorphe Abbildung ist, ist $\Pi_{1}(M)$ nach dem Remmertschen Abbildungssatz [4, Satz 23] eine analytische Menge in $X_{1}$. Da $X_{1}$ irreduzibel ist, und da $\Pi_{1}(M)$ einene offenen Teil von $X_{1}$ enthält, ist $\Pi_{1}(M)=X_{1}$. Hieraus entnimmt man, dass $\Pi_{1}: M \rightarrow X_{1}$ und $\Pi_{2}: M \rightarrow X_{2}$ holomorphe Überlagerungsabbildungen sind, und $M$ ist folglich der Graph der irreduziblen holomorphen Überlagerungskorrespondenz

$$
\Pi_{2} \circ \Pi_{1}^{-1}: X_{1} \rightarrow_{k} X_{2} \text {. }
$$

Ist $K \cong X_{1}$ eine kompakte Menge, so ist auch $\Pi_{1}{ }^{-1}(K) \cong X_{1} \times X_{2}$ kompakt. Nun schneidet aber $\Pi_{1}^{-1}(K)$ sämtliche irreduziblen Komponenten von $\mathscr{G}[F]$. Die Anzahl der irreduziblen Komponenten des Graphen ist also endlich, und jede Komponente ist Graph einer irreduziblen holomorphen Überlagerungskorrespondenz.

Proposition 3. Es seien $X_{1}$ und $X_{2}$ irreduzible komplexe Räume, $X_{1}$ normal, und $F: X_{1} \rightarrow_{k} X_{2}$ eine irreduzible holomorphe Überlagerungskorrespondenz. Dann gibt es zu jeder in $X_{2}$ holomorphen Funktion $g$ ein irreduzibles Polynom

$$
P\left(\zeta ; x_{1}\right)=\zeta^{n}+\sum_{p=0}^{n-1} \zeta^{p} A_{p}\left(x_{1}\right)
$$

über dem Ring $\mathcal{O}\left(X_{1}\right)$ der in $X_{1}$ holomorphen Funktionen derart, dass

$$
P\left(g\left(x_{2}\right) ; x_{1}\right)=0
$$

für jedes Punktpaar $\left(x_{1}, x_{2}\right) \in X_{1} \times X_{2}$ mit $x_{2} \in F\left(x_{1}\right)$. Ist die Funktion $g$ in $X_{2}$ beschränkt, so sind die Koeffizientenfunktionen $A_{p}$ in $X_{1}$ beschränkt.

BEwEIS. Es ist $\Pi_{1} \circ \mu: \mathscr{G}\left[F^{*}\right]^{*} \rightarrow X_{1}$ eine holomorphe Überlagerungsabbildung, wenn $\mu: \mathscr{G}[F]^{*} \rightarrow \mathscr{G}[F]$ eine Normalisierung des Graphen $\mathscr{G}[F]$ bezeichnet. Die Behauptung ergibt sich dann durch Anwendung eines Satzes der Theorie der analytischen Überlagerungen $[1, \S 3, \S 11]$ auf die in $\mathscr{G}[F]^{*}$ holomorphe Funktion $g \circ \Pi_{2} \circ \mu$.

Sind $X_{1}$ und $X_{2}$ Gebiete des $C^{n}$, so lässt sich Proposition 3 auf die komplexen Koordinaten in $X_{2}$ anwenden. Die Korrespondenz wird dann in einem gewissen Sinne als "algebroide Abbildung" dargestellt.

2.

Für die späteren Úberlegungen brauchen wir folgendes Lemma über Pseudopolynome: 
Lemma. Es sei $A$ ein Gebiet des $\boldsymbol{C}^{n}(\boldsymbol{z}), \boldsymbol{z}=\left(z_{1}, \ldots, z_{n}\right)$, und

$$
P(\zeta ; z)=\zeta^{m}+\sum_{\mu=0}^{m-1} \zeta^{\mu} B_{\mu}(z)
$$

ein über dem Ring $\mathcal{O}(A)$ der in $A$ holomorphen Funktionen irreducibles Polynom. Ist die Resultante

$$
\mathscr{R}\left(P(\zeta ; z), \frac{\partial}{\partial z_{1}} P(\zeta ; z)\right)
$$

identisch 0 in $A$, so sind die Koeffizienten $B_{\mu}$ sämtlich von $z_{1}$ unabhängig.

(Bei der Resultantenbildung wird $\partial P / \partial z_{1}$ als Polynom $m$-ten Grades mit höchstem Koeffizient 0 betrachtet.)

BEweIs. Es sei $M \cong A$ die Nullstellenmenge der Diskriminante von $P$ und $U$ eine offene einfach zusammenhängende Teilmenge von $A \backslash M$. Dann gibt es $m$ verschiedene in $U$ holomorphe Funktionen $\zeta_{1}(z), \ldots$, $\zeta_{m}(z)$ derart, dass

in $U$. Es gilt also

$$
P(\zeta ; z)=\prod_{\mu=1}^{m}\left(\zeta-\zeta_{\mu}(z)\right)
$$

$$
\frac{\partial}{\partial z_{1}} P(\zeta ; z)=-\sum_{\mu=1}^{m}\left\{\frac{\partial}{\partial z_{1}} \zeta_{\mu}(z) \cdot \prod_{\nu \neq \mu}\left(\zeta-\zeta_{\nu}(z)\right)\right\}
$$

in $U$. Aus der Voraussetzung folgt, dass $P$ und $\partial P / \partial z_{1}$ eine gemeinsame Wurzel $\zeta_{\nu}(z)$ in $U$ haben, und für diese gilt offenbar

$$
\frac{\partial}{\partial z_{1}} \zeta_{\nu}(z) \equiv 0
$$

in $U$. Diese Funktion lässt sich in $A \backslash M$ unbeschränkt analytisch fortsetzen, und dadurch, weil $P$ irreduzibel in $A$ ist, in jede andere Wurzel von $P$ überführen. Bei der Fortsetzung bleibt die partielle Ableitung nach $z_{1}$ identisch 0 . Die Wurzeln und damit die Koeffizienten von $P$ sind also in $A \backslash M$ von $z_{1}$ unabhängig, und daraus folgt die Behauptung des Lemmas.

\section{3.}

Es sei $A \leqq C^{n}(z), z=\left(z_{1}, \ldots, z_{n}\right)$, ein beschränktes Gebiet. Wir sagen, dass $A$ einfachen Rand hat, wenn jede holomorphe Abbildung $f: B \rightarrow C^{n}$ mit $f(B) \cong \partial A$, wo $B$ ein Gebiet der komplexen Ebene ist, konstant ist.

Wir können jetzt folgenden Satz beweisen. (Für die Definition der Verwandtschaft im engeren Sinne vgl. [8, S. 298].) 
SATZ 1. Es seien

und

$$
\left.A_{j} \leqq C^{m_{j}}{ }_{j} z\right), \quad{ }_{j} z=\left({ }_{j} z_{1}, \ldots,{ }_{j} z_{m_{j}}\right), \quad j=1, \ldots, s,
$$

$$
B_{k} \subseteq \boldsymbol{C}^{n_{k}}\left({ }_{k} \boldsymbol{w}\right), \quad{ }_{k} \boldsymbol{w}=\left({ }_{k} w_{1}, \ldots,{ }_{k} w_{n_{k}}\right), \quad k=1, \ldots, t,
$$

beschränkte Gebiete mit einfachen Rändern, $N=\sum m_{j}=\sum n_{k}$, und $F: A \rightarrow_{k} B$, wo $A=A_{1} \times \ldots \times A_{s}$ und $B=B_{1} \times \ldots \times B_{t}$, eine irreduzible holomorphe Überlagerungskorrespondenz. Dann gibt es eine bijektive Abbildung

$$
\sigma:\{1, \ldots, s\} \rightarrow\{1, \ldots, t\}
$$

derart, dass die Abbildungen

$$
p_{j} \circ \Pi_{1}: \mathscr{G}[F] \rightarrow A_{j} \quad \text { und } \quad q_{\sigma(j)} \circ \Pi_{2}: \mathscr{G}[F] \rightarrow B_{\sigma(j)}
$$

für $j=1, \ldots, s$ im engeren Sinne verwandt sind. Hierbei sind $p_{j}: A \rightarrow A_{j}$, $q_{k}: B \rightarrow B_{k}$ die natürlichen Projektionen und $\Pi_{1}: \mathscr{G}[F] \rightarrow A, \Pi_{2}: \mathscr{G}[F]$ $\rightarrow B$ die Restriktionen der natürlichen Projektionen $A \times B \rightarrow A, A \times B \rightarrow B$.

BeweIs. Für $s=t=1$ ist nichts zu beweisen. Auf Grund der Symmetrie zwischen $A$ und $B$ in dem Problem können wir dann $s \geqq t$ und $s \geqq 2$ annehmen. Nach Proposition 3 gibt es Polynome $P_{k}{ }^{\nu}, k=1, \ldots, t$, $v=1, \ldots, n_{k}$, mit in $A$ holomorphen und beschränkten Koeffizienten derart, dass jedes $P_{k}{ }^{v}$ über $\mathcal{O}(A)$ irreduzibel ist, und in jedem Punkt $(\boldsymbol{z}, \boldsymbol{w})$ mit $\boldsymbol{w} \in F(\boldsymbol{z})$

gilt.

$$
P_{k}{ }_{k}\left({ }_{k} w_{v} ; z\right)=0, \quad k=1, \ldots, t, v=1, \ldots, n_{k},
$$

Sei $a_{1}, a_{2}, \ldots$ eine Punktfolge in $A_{1}$, die gegen einen Randpunkt von $A_{1}$ konvergiert. Für jedes $(k, v)$ bildet jede Koeffizientenfolge in

$$
P_{k}{ }^{\nu}\left({ }_{k} w_{v} ; \boldsymbol{a}_{p},{ }_{2} z, \ldots,{ }_{s} z\right), \quad p=1,2, \ldots,
$$

nach Montel eine normale Familie in $A_{2} \times \ldots \times A_{s}$. Es gibt also eine Teilfolge $\boldsymbol{b}_{1}, \boldsymbol{b}_{2}, \ldots$ der Folge $\left(\boldsymbol{a}_{p}\right)$ derart, dass sämtliche Koeffizientenfolgen in $A_{2} \times \ldots \times A_{s}$ kompakt konvergieren. Die Grenzpolynome seien $\widetilde{P}_{k}{ }^{\nu}\left({ }_{k} w_{\nu} ;{ }_{2} z, \ldots,{ }_{s} z\right)$.

Es sei $\left({ }_{2} z, \ldots,{ }_{s} z\right) \in A_{2} \times \ldots \times A_{s}$ beliebig aber fest, und es sei $\boldsymbol{c}_{p} \in F\left(\boldsymbol{b}_{p},{ }_{2} z, \ldots,{ }_{s} z\right)$. Da $B$ beschränkt ist, hat die Folge $\left(\boldsymbol{c}_{p}\right)$ mindestens einen Häufungspunkt $\boldsymbol{w}$. Wegen der Eigentlichkeit der Abbildung $\Pi_{2}$ liegt $w$ auf dem Rand $\partial B$ von $B$. Hieraus folgt, dass es zu jedem Punkt $\left({ }_{2} z, \ldots,{ }_{s} z\right) \in A_{2} \times \ldots \times A_{s}$ einen Punkt $\left({ }_{1} w, \ldots,{ }_{t} w\right)$ auf dem Rand von $B$ derart gibt, dass

$$
\tilde{P}_{k}{ }_{k}\left({ }_{k} w_{v} ;{ }_{2} z, \ldots,{ }_{s} z\right)=0
$$

für $k=1, \ldots, s, \nu=1, \ldots, n_{k}$. 
Wir können eine offene Teilmenge $U \subseteq A_{2} \times \ldots \times A_{s}$ so wählen, dass jedes Polynom $\tilde{P}_{k}{ }^{v}$ in $U$ vollständig zerfällt:

$$
\tilde{P}_{k}{ }^{\nu}\left({ }_{k} w_{v} ;{ }_{2} z, \ldots,{ }_{s} z\right)=\prod_{\mu}\left({ }_{k} w_{\nu}-{ }_{k} \zeta_{\nu}{ }^{(\mu)}\left({ }_{2} z, \ldots,{ }_{s} z\right)\right)
$$

mit in $U$ holomorphen Funktionen ${ }_{k} \zeta_{v}^{(\mu)}$. Zu jedem Punkt $\left({ }_{2} z, \ldots,{ }_{s} z\right) \in U$ gibt es dann eine Indexkombination $\left(\mu_{k v}\right)$ derart, dass der Punkt $\left({ }_{k} \zeta_{v}{ }^{\left(\mu_{k v}\right)}\left({ }_{2} z, \ldots, g_{s} z\right)\right)$ auf dem Rand von $B$ liegt. Hieraus folgt, dass eine offene Teilmenge $V$ von $U$, eine Zahl $l, 1 \leqq l \leqq t$, und eine Indexkombination $\mu_{1}, \ldots, \mu_{n_{l}}$ derart existieren, dass für alle $\left({ }_{2} z, \ldots,{ }_{s} z\right)$ in einer in $V$ dichten Teilmenge

$$
\left({ }_{l} \zeta_{v}^{\left(\mu_{p}\right)}\left({ }_{2} z, \ldots,{ }_{s} z\right)\right) \in \partial B_{l}
$$

gilt. Da $B_{l}$ einfachen Rand hat, folgt hieraus, dass die Funktionen $\zeta_{\nu}{ }^{\left(\mu_{v}\right)}, \nu=1, \ldots, n_{l}$, alle konstant sind, also dass jedes der Polynome $\widetilde{P}_{l}^{1}, \ldots, \tilde{P}_{l}^{n_{l}}$ eine konstante Wurzel besitzt. Es gilt dann für $k=1, \ldots, n_{l}$ und jedes $(\mu, v)$ mit $\mu \geqq 2,1 \leqq \nu \leqq m_{\mu}$, dass

$$
\mathscr{R}\left(\tilde{P}_{l}^{k}, \frac{\partial}{\partial_{\mu} z_{\nu}} \tilde{P}_{l}^{k}\right)
$$

identisch 0 in $A_{2} \times \ldots \times A_{s}$ ist. Mittels des Weierstrass'schen Satzes folgt dann, dass

$$
\mathscr{R}\left(P_{l}{ }_{l}\left({ }_{l} w_{k} ; \boldsymbol{b}_{p},{ }_{2} z, \ldots,{ }_{s} z\right), \frac{\partial}{\partial_{\mu} z_{v}} P_{l}{ }_{l}\left({ }_{l} w_{k} ; \boldsymbol{b}_{p},{ }_{2} z, \ldots,{ }_{s} z\right)\right)
$$

für $p \rightarrow \infty$ in $A_{2} \times \ldots \times A_{s}$ kompakt gegen 0 konvergiert, $k=1, \ldots, n_{l}$, $\mu, v$ beliebig mit $\mu \geqq 2$ und $1 \leqq \nu \leqq m_{\mu}$. Für jede Indexkombination $\left(\mu_{j}, v_{j}\right), j=1, \ldots, t$, mit $\mu_{j} \geqq 2$ und $1 \leqq \nu_{j} \leqq m_{\mu_{j}}$, und jede Indexkombination $\left(k_{j}\right), 1 \leqq k_{j} \leqq n_{j}, j=1, \ldots, t$, ist

$$
\Phi(z)=\prod_{j=1}^{t} \mathscr{R}\left(P_{j}^{k_{j}}, \frac{\partial}{\partial_{\mu_{j}} z_{v_{j}}} P_{j}^{k_{j}}\right)
$$

eine in $A$ holomorphe Funktion. Es ist aber $\Phi$ identisch 0. Diese Aussage folgt daraus, dass für jede Folge $a_{1}, a_{2}, \ldots$ in $A_{1}$, die gegen einen Randpunkt von $A_{1}$ konvergiert, die Folge

$$
\Phi\left(a_{p},{ }_{2} z, \ldots, s_{s} z\right), \quad p=1,2, \ldots,
$$

in $A_{2} \times \ldots \times A_{s}$ kompakt gegen 0 konvergiert. Jede Folge $\left(a_{p}\right)$ enthält nämlich eine Teilfolge $\left(b_{p}\right)$, für welche dies gilt. (Die Zahl $l$ ist von der Folge $\left(b_{p}\right)$ abhängig; bei der Bildung der Funktionen $\Phi$ haben wir aber über alle $l$ multipliziert.) 
Es gibt dann eine Zahl $\sigma(1), 1 \leqq \sigma(1) \leqq t$, so dass die Resultanten

$$
\mathscr{R}\left(P_{\sigma(1)}^{k}, \frac{\partial}{\partial_{\mu} z_{\nu}} P_{\sigma(1)}^{k}\right)
$$

für $k=1, \ldots, n_{\sigma(1)}, \mu \geqq 2$ und $1 \leqq \nu \leqq m_{\mu}$ alle identisch verschwinden. Da $P_{\sigma(1)}^{k}, k=1, \ldots, n_{\sigma(1)}$, über $\mathcal{O}(A)$ irreduzibel ist, folgt dann mit Hilfe des obigen Lemmas, dass die Koeffizienten der Polynome $P_{\sigma(1)}^{k}$ nur von ${ }_{1} z$ abhängig sind. Die Abbildung $q_{\sigma(1)} \circ \Pi_{2}$ ist also von der Abbildung $p_{1} \circ \Pi_{1}$ strikt abhängig.

Es sei $a_{1}, a_{2}, \ldots$ eine Punktfolge aus $A_{2}$, die gegen einen Randpunkt von $A_{2}$ konvergiert. Ist

$$
\left({ }_{1} z,{ }_{3} z, \ldots,{ }_{s} z\right) \in A_{1} \times A_{3} \times \ldots \times A_{s}
$$

ein fester Punkt und

$$
\left({ }_{1} w^{(p)}, \ldots,{ }_{t} w^{(p)}\right) \in F\left({ }_{1} z, a_{p},{ }_{3} z, \ldots,{ }_{s} z\right),
$$

so kann die Folge ${ }_{\sigma_{(1)}} \boldsymbol{w}^{(p)}, p=1,2, \ldots$, wegen der oben bewiesenen strikten Abhängigkeit keinen Häufungspunkt auf dem Rand von $B_{\sigma(1)}$ besitzen. Da $\Pi_{2}$ eigentlich ist, muss es also noch ein Faktorgebiet in $B$ geben. Wir können jetzt die obigen Schlüsse mit Folgen aus $A_{2}$ durchführen. Hierbei braucht man aber nur die Polynome zu betrachten, die zu Gebieten $B_{k}$ mit $k \neq \sigma(1)$ gehören. Es folgt dann, dass es eine Zahl $\sigma(2), 1 \leqq \sigma(2) \leqq t$, $\sigma(2) \neq \sigma(1)$, derart gibt, dass die Abbildung $q_{\sigma(2)} \circ \Pi_{2}$ von der Abbildung $p_{2} \circ \Pi_{1}$ strikt abhängig ist.

Diese Betrachtung lässt sich anwenden, bis es keine Gebiete $A_{j}$ mehr gibt. Daher gilt $s=t$, und es gibt eine bijektive Abbildung

$$
\sigma:\{1, \ldots, s\} \rightarrow\{1, \ldots, t\}
$$

derart, dass für $j=1, \ldots, s$ die Abbildung $q_{\sigma(j)} \circ \Pi_{2}$ von der Abbildung $p_{j} \circ \Pi_{1}$ strikt abhängig ist.

Da $s=t$, ist die Schlussweise auch auf $F^{-1}$ anwendbar. Es gibt also eine bijektive Abbildung

$$
\tau:\{1, \ldots, t\} \rightarrow\{1, \ldots, s\}
$$

derart, dass für $k=1, \ldots, t$ die Abbildung $p_{\tau(k)} \circ \Pi_{1}$ von der Abbildung $q_{k} \circ \Pi_{2}$ strikt abhängig ist. Da die Abbildungen $p_{i} \circ \Pi_{1}$ und $p_{j} \circ \Pi_{1}$ für $i \neq j$ unabhängig sind, folgt dann, dass $\tau=\sigma^{-1}$ und damit die Behauptung des Satzes.

Corollar: $E s$ ist $m_{j}=n_{\sigma(j)}$ für $j=1, \ldots, s$.

Dies folgt sofort daraus, dass $p_{j} \circ \Pi_{1}$ den lokalen Rang $m_{j}$ und $q_{k} \circ \Pi_{2}$ den lokalen Rang $n_{k}$ hat. 
Es ist wohlbekannt, dass eine Hyperkugel des $\boldsymbol{C}^{n}$ für jedes $n$ ein Gebiet mit einfachem Rand ist. Satz 1 lässt sich also auf direkte Produkte von Hyperkugeln anwenden. Als Spezialfälle erhalten wir für $\boldsymbol{C}^{2}$ die folgenden beiden Aussagen, von denen die erste von K. Stein bewiesen wurde (unveröffentlicht):

Es gibt keinen komplexen Raum, der sich durch holomorphe Überlagerungsabbildungen sowohl auf eine Hyperkugel als auch auf einen Dizylinder des $\boldsymbol{C}^{2}$ abbilden lässt.

Es gibt keinen komplexen Raum, auf welchen sich sowohl eine Hyperkugel als auch ein Dizylinder des $\boldsymbol{C}^{2}$ durch holomorphe Überlagerungsabbildungen abbilden lässt.

4.

Jetzt wollen wir analytische Polyedergebiete des $\boldsymbol{C}^{n}$ betrachten. Für die Definitionen der eingehenden Begriffe wird auf [5] und [6] verwiesen.

SATZ 2. Es seien $A_{1} \leqq C^{n}(z), \quad z=\left(z_{1}, \ldots, z_{n}\right)$, und $A_{2} \leqq C^{n}(w), \quad w=$ $\left(w_{1}, \ldots, w_{n}\right)$, analytische Polyedergebiete der Stufe $s_{1}$ bzw. $s_{2}$, und $F: A_{1} \rightarrow_{k} A_{2}$ eine irreduzible holomorphe Überlagerungskorrespondenz. Dann induziert $F$ eine bijektive Zuordnung zwischen den charakteristischen Zerlegungen von $A_{1}$ und $A_{2}$ derart, dass die Abbildungen $f \circ \Pi_{1}: \mathscr{G}[F] \rightarrow \boldsymbol{C}$ und $g \circ \Pi_{2}: \mathscr{G}[F] \rightarrow C$ im engeren Sinne verwandt sind, wenn $f$ und $g$ Zerlegungsfunktionen auf $A_{1}$ bzw. $A_{2}$ für zugeordnete Zerlegungen sind.

Beweis: Auf Grund der Symmetrie zwischen $A_{1}$ und $A_{2}$ im Problem können wir $s_{1} \geqq s_{2}$ annehmen. Es sei $\left(f_{1}, \ldots, f_{s_{1}}, \ldots, f_{r}\right)$ ein Minimalsystem für $A_{1}$ derart, dass $f_{1}, \ldots, f_{s_{1}}$ die charakteristischen Zerlegungen von $A_{1}$ erzeugen, und $\left(g_{1}, \ldots, g_{s_{2}}, \ldots, g_{t}\right)$ ein entsprechendes System für $A_{2}$. Wir wollen dann zeigen, dass es eine injektive (also bijektive) Abbildung

$$
\sigma:\left\{1, \ldots, s_{1}\right\} \rightarrow\left\{1, \ldots, s_{2}\right\}
$$

derart gibt, dass $f_{j} \circ \Pi_{1}$ und $g_{\sigma(j)} \circ \Pi_{2}$ für $j=1, \ldots, s_{1}$ im engeren Sinne verwandt sind. Dies wird durch Induktion bewiesen. Wir nehmen also an, dass $\sigma(1), \ldots, \sigma(k-1)$ schon gefunden sind, so dass die Bedingungen erfüllt sind, und wollen $\sigma(k)$ finden.

Da $\left(f_{1}, \ldots, f_{r}\right)$ ein Minimalsystem von $A_{1}$ ist, gibt es einen Punkt $a \in \partial A_{1}$, für welchen $\left|f_{k}(\boldsymbol{a})\right|=1$ und $\left|f_{v}(\boldsymbol{a})\right|<1$ für $\nu \neq k$. Da $f_{k}$ nicht konstant ist, kann man ausserdem $a$ so wählen, dass eine partielle Ableitung, zum Beispiel $\partial f_{k} / \partial z_{1}$, von $f_{k}$ in $a$ nicht verschwindet. Das Funktionensystem 


$$
\begin{aligned}
\tilde{z}_{1} & =f_{k}\left(z_{1}, \ldots, z_{n}\right) \\
\tilde{z}_{2} & =z_{2} \\
& \vdots \\
\tilde{z}_{n} & =z_{n}
\end{aligned}
$$

hat dann eine in $\boldsymbol{a}$ nicht verschwindende Funktionaldeterminante und definiert also eine biholomorphe Abbildung $\varphi$ einer offenen Umgebung $U$ von $\boldsymbol{a}$ auf einen Polyzylinder

$$
D=D_{1} \times \ldots \times D_{n}
$$

in $C^{n}(\tilde{z})$. Wählen wir $U$ so klein, dass $\left|f_{v}\right|<1$ für $v \neq k$ in $U$, so gilt

wo

und

$$
\varphi(A \cap U)=D^{\prime}=D_{1}^{\prime} \times D^{\prime \prime},
$$

$$
D_{1}^{\prime}=D_{1} \cap\left\{\tilde{z}_{1}|| \tilde{z}_{1} \mid<1\right\}
$$

$$
D^{\prime \prime}=D_{2} \times \ldots \times D_{n} \cong C^{n-1}\left(\tilde{z}_{\dot{2}}, \ldots, \tilde{z}_{n}\right) .
$$

Nach Proposition 3 gibt es über $\mathcal{O}\left(A_{1}\right)$ irreduzible Polynome $P_{\lambda}, \lambda=1, \ldots, t$, so dass

$$
P_{\lambda}\left(g_{\lambda}(\boldsymbol{w}) ; z\right)=0 \quad \text { für } \lambda=1, \ldots, t,
$$

wenn $\boldsymbol{w} \in F(\boldsymbol{z})$. Diese Polynome brauchen nicht über dem Ring $\mathcal{O}\left(U \cap A_{1}\right)$ irreduzibel zu sein. Es gibt aber Polynome $Q_{\lambda}$ über $\mathcal{O}\left(U \cap A_{1}\right)$, so dass $Q_{\lambda}, \lambda=1, \ldots, t$, in $U \cap A_{1}$ ein irreduzibler Teiler von $P_{\lambda}$ ist, und so dass jeder Punkt $\boldsymbol{z} \in U \cap A_{1}$ einen Bildpunkt $\boldsymbol{w}$ bei $\boldsymbol{F}$ hat, für den $Q_{\lambda}\left(g_{\lambda}(\boldsymbol{w}) ; \boldsymbol{z}\right)=0$ für $\lambda=1, \ldots, t$. Wir nennen $\boldsymbol{w}$ einen ausgezeichneten Bildpunkt von $\boldsymbol{z}$ bezüglich $\left(Q_{\lambda}\right)$.

Diese Aussage wird so bewiesen:

Es gibt eine mindestens 1-codimensionale analytische Menge $M \cong A_{1}$ derart, dass die Abbildung

$$
\Pi_{1}: \mathscr{G}\left[F^{\prime}\right] \backslash \Pi_{1}^{-1}(M) \rightarrow A_{1} \backslash M
$$

lokaltopologisch ist. (Als $M$ wählen wir die Vereinigung einer kritischen Menge der analytischen Utberlagerung $\Pi_{1} \circ \mu: \mathscr{G}[F]^{*} \rightarrow A_{1}$ und des Bildes der Menge der nicht gewöhnlichen Punkte von $\mathscr{G}\left[F^{\prime}\right]$ bei $\Pi_{1}$.) Es sei $V$ eine einfach zusammenhängende offene Teilmenge von $\left(U \cap A_{1}\right) \backslash M$. Es gibt dann eine holomorphe Abbildung $\psi: V \rightarrow \mathscr{G}[F]$ derart, dass

$$
\Pi_{1} \circ \psi: V \rightarrow \Pi_{1}^{-1}(V) \rightarrow V
$$

die identische Abbildung der Menge $V$ ist. Die in $V$ holomorphe Funktion

$$
\zeta_{\lambda}(z)=g_{\lambda} \circ \Pi_{2} \circ \psi(z)
$$


ist dann für $\lambda=1, \ldots, t$ eine Wurzel des Polynoms $P_{\lambda}$. Jede Funktion $\zeta_{\lambda}$ lässt sich unbeschränkt in $\left(U \cap A_{1}\right) \backslash M$ analytisch fortsetzen. Man zeigt dann mit der üblichen Methode, dass für jedes $\lambda$ die Funktion $\zeta_{\lambda}$ und ihre Fortsetzungen genau die Wurzeln eines über $\mathcal{O}\left(U \cap A_{1}\right)$ irreduziblen Faktorpolynoms $Q_{\lambda}$ des Polynoms $P_{\lambda}$ bilden. Simultane analytische Fortsetzung sämtlicher Funktionen $\zeta_{\lambda}$ von einem Punkt von $V$ aus ergibt, dass jeder Punkt der Menge $\left(U \cap A_{1}\right) \backslash M$ einen bezüglich $\left(Q_{\lambda}\right)$ augezeichneten Bildpunkt besitzt. Ist $z^{(0)} \in U \cap A_{1} \cap M$, so ist es möglich eine Punktfolge $\left(z^{(p)}\right)$ der Menge $\left(U \cap A_{1}\right) \backslash M$ derart zu wählen, dass $z^{(p)}$ für $p \rightarrow \infty$ gegen $z^{(0)}$ konvergiert. Es sei $\left(w^{(p)}\right)$ eine Folge ausgezeichneter Bildpunkte. Da $\Pi_{1}$ eigentlich ist, hat die Folge $\left(w^{(p)}\right)$ einen Häufungspunkt $w^{(0)} \in A_{2}$, der offenbar ein bezüglich $\left(Q_{\lambda}\right)$ ausgezeichneter Bildpunkt des Punktes $z^{(0)}$ ist.

Damit ist die Hilfsaussage bewiesen.

Vermöge der Abbildung $\varphi^{-1}$ werden die Koeffizienten der Polynome $Q_{\lambda}$ Funktionen von $\tilde{z}$ in $D^{\prime}$. Es sei $\tilde{a}_{1}, \tilde{a}_{2}, \ldots$ eine Folge in $D_{1}^{\prime}$ mit $\left|\tilde{a}_{p}\right| \rightarrow 1$ für $p \rightarrow \infty$. Für jede Koeffizientenfunktion $H(\tilde{z})$ eines der Polynome $Q_{\lambda}$ ist die Folge $H\left(a_{p}, \tilde{z}_{2}, \ldots, \tilde{z}_{n}\right)$ dann nach Montel eine normale Familie in $D^{\prime \prime}$. Es gibt also eine Teilfolge $\left(\tilde{b}_{p}\right)$ der Folge $\left(\tilde{a}_{p}\right)$ derart, dass jeder Koeffizient der Polynome $Q_{\lambda}$ für diese Folge in $D^{\prime \prime}$ kompakt konvergiert, das heisst es gibt Polynome $\widetilde{Q}_{\lambda}$ über $\mathcal{O}\left(D^{\prime \prime}\right)$, so dass für $p \rightarrow \infty$

$$
Q_{\lambda}\left(\zeta_{\lambda} ; \tilde{b}_{p}, \tilde{z}_{2}, \ldots, \tilde{z}_{n}\right) \rightarrow \widetilde{Q}_{\lambda}\left(\zeta_{\lambda} ; \tilde{z}_{2}, \ldots, \tilde{z}_{n}\right)
$$

kompakt in $D^{\prime \prime}$. Wir behaupten jetzt, dass es für jedes $\left(\tilde{z}_{2}{ }^{(0)}, \ldots, \tilde{z}_{n}{ }^{(0)}\right) \in$ $D^{\prime \prime}$ ein $\lambda, 1 \leqq \lambda \leqq t$, derart gibt, dass das Polynom

$$
\widetilde{Q}_{\lambda}\left(\zeta_{\lambda} ; \tilde{z}_{2}{ }^{(0)}, \ldots, \tilde{z}_{n}{ }^{(0)}\right)
$$

eine Wurzel $\zeta_{\lambda}$ mit $\left|\zeta_{\lambda}\right|=1$ hat. Sei nämlich $\left(\boldsymbol{w}^{(p)}\right)$ eine bezüglich $\left(Q_{\lambda}\right)$ ausgezeichnete Bildpunktfolge der Folge

$$
\varphi^{-1}\left(\tilde{b}_{p}, \tilde{z}_{2}{ }^{(0)}, \ldots, \tilde{z}_{n}{ }^{(0)}\right), \quad p=1,2, \ldots,
$$

und $\boldsymbol{w}^{(0)}$ ein Häufungspunkt der Folge $\left(\boldsymbol{w}^{(p)}\right)$. Wegen der Eigentlichkeit der Abbildung $\Pi_{2}$ liegt $w^{(0)}$ dann auf dem Rand von $A_{2}$; das heisst, es gibt ein $\lambda$ mit $\mid g_{\lambda}\left(\boldsymbol{w}^{(0)} \mid=1\right.$.

Ferner gilt offenbar

$$
\tilde{Q}_{\lambda}\left(g_{\lambda}\left(\boldsymbol{w}^{(0)}\right) ; \tilde{z}_{2}^{(0)}, \ldots, \tilde{z}_{n}^{(0)}\right)=0 .
$$

Hieraus folgt, dass es ein $\lambda_{0}$ gibt, so dass $\widetilde{Q}_{\lambda_{0}}$ in $D^{\prime \prime}$ eine konstante Wurzel besitzt. Es ist also 


$$
\mathscr{R}\left(\widetilde{Q}_{\lambda_{0}}, \frac{\partial}{\partial \tilde{z}_{\mu}} \widetilde{Q}_{\lambda_{0}}\right)=0
$$

identisch in $D^{\prime \prime}$ für $\mu=2, \ldots, n$. Hieraus folgt nach Weierstrass, dass für jedes $\mu=2, \ldots, n$ die Resultante

$$
\mathscr{R}\left(Q_{\lambda_{0}}\left(\zeta_{\lambda_{0}} ; \tilde{b}_{p}, \tilde{z}_{2}, \ldots, \tilde{z}_{n}\right), \frac{\partial}{\partial \tilde{z}_{\mu}} Q_{\lambda_{0}}\left(\zeta_{\lambda_{0}} ; \tilde{b}_{p}, \tilde{z}_{2}, \ldots, \tilde{z}_{n}\right)\right)
$$

für $p \rightarrow \infty$ kompakt in $D^{\prime \prime}$ gegen 0 konvergiert. Für jede Indexkombination $\mu_{\lambda}, \lambda=1, \ldots, t, 2 \leqq \mu_{\lambda} \leqq n$, ist folglich

$$
\Phi(\tilde{z})=\prod_{\lambda=1}^{t} \mathscr{R}\left(Q_{\lambda}, \frac{\partial}{\partial \tilde{z}_{\mu_{\lambda}}} Q_{\lambda}\right)
$$

eine in $D^{\prime}$ holomorphe Funktion, die für $\left(\tilde{z}_{2}, \ldots, \tilde{z}_{n}\right)$ beliebig, aber fest, in $D^{\prime \prime}$ in sämtlichen Randpunkten des Gebietes $D_{1}^{\prime}$ mit $\left|\tilde{z}_{1}\right|=1$ den Randwert 0 hat. Die Funktionen $\Phi$ sind also alle identisch 0 in $D^{\prime}$. Es gibt folglich eine Zahl $\lambda$, so dass alle Resultanten

$$
\mathscr{R}\left(Q_{\lambda}, \frac{\partial}{\partial \tilde{z}_{\mu}} Q_{\lambda}\right), \quad \mu=2, \ldots, n,
$$

in $D^{\prime}$ identisch verschwinden. Da das Polynom $Q_{\lambda}$ über $\mathcal{O}\left(D^{\prime}\right)$ irreduzibel ist, folgt aus dem Lemma, dass die Koeffizienten von $Q_{\lambda}$ nur von $\tilde{z}_{1}$ abhängen, mit anderen Worten, dass jeder Koeffizient von $Q_{\lambda}$ strikt von $f_{k}$ abhängig ist.

Es sei $M^{\prime} \cong A_{1}$ die Nullstellenmenge der Diskriminante des Polynoms $P_{\lambda}$ und $V^{\prime}$ eine einfach zusammenhängende offene Teilmenge der Menge $\left(U \cap A_{1}\right) \backslash M^{\prime}$. Es gibt also dann in $V^{\prime}$ eine von $f_{k}$ strikt abhängige holomorphe Funktion $\zeta(z)$, die Wurzel von $P_{\lambda}$ ist. Sie lässt sich in $A_{1} \backslash M^{\prime}$ beliebig analytisch fortsetzen und bleibt dabei lokal strikt von $f_{k}$ abhängig. Da $\zeta$ sich wegen der Irreduzibilität des Polynomes $P_{\lambda}$ über $\mathcal{O}\left(A_{1}\right)$ durch analytische Fortsetzung in $A_{1} \backslash M^{\prime}$ in jede andere Wurzel von $P_{\lambda}$ überführen lässt, sind alle Wurzeln, und damit die Koeffizienten von $P_{\lambda}$, strikt von $f_{k}$ abhängig. Die Abbildung $g_{\lambda} \circ \Pi_{2}$ ist also von der Abbildung $f_{k} \circ \Pi_{1}$ strikt abhängig. Da beide Abbildungen überall in $\mathscr{G}[F]$ den lokalen Rang 1 haben, sind sie sogar im engeren Sinne verwandt. Die Funktion $g_{\lambda}$ ist mit einer der Funktionen $g_{1}, \ldots, g_{s_{2}}$ im engeren Sinne verwandt, und es gibt also eine Zahl $\sigma(k), 1 \leqq \sigma(k) \leqq s_{2}$, so dass die Abbildungen $f_{k} \circ \Pi_{1}$ und $g_{\sigma(k)} \circ \Pi_{2}$ im engeren Sinne verwandt sind.

Für $k=1$ haben wir damit den Induktionsanfang bewiesen. Für $k>1$ haben wir noch zu zeigen, dass $\sigma(k) \neq \sigma(j)$ für $1 \leqq j<k$. Dies folgt aber sofort daraus, dass die Abbildungen $f_{j} \circ \Pi_{1}$ und $f_{k} \circ \Pi_{1}$ unabhängig sind.

Damit ist der Satz bewiesen. 
Speziell kann $F$ eine holomorphe Überlagerungsabbildung sein. Aus Satz 2 erhält man dann den Remmert--Steinschen Satz [5, Satz 14] für Polyedergebiete beliebiger Dimension mit der vom Verfasser [6] bewiesenen Verschärfung.

Der Satz lässt sich zum Beispiel auf euklidische Polyedergebiete [5, S. 186] anwenden. Als Verallgemeinerung des Satzes 16 von [5] haben wir:

SATz 3. Es seien $A_{1} \cong \boldsymbol{C}^{n}\left(z_{1}, \ldots, z_{n}\right)$ und $A_{2} \cong \boldsymbol{C}^{n}\left(w_{1}, \ldots, w_{n}\right), n \geqq 2$, euklidische Polyedergebiete mit mindestens $n+1$ affinen Zerlegungen, von denen je $n$ unabhängig sind. Dann ist jede irreduzible holomorphe Überlagerungskorrespondenz $F: A_{1} \rightarrow_{k} A_{2}$ die Restriktion einer affinen Abbildung.

BEwEIs. Wir dürfen annehmen (eventuell sind lineare Koordinatentransformationen auszuführen), dass die Funktionen $z_{1}, \ldots, z_{n}$ und $z_{1}+\ldots+z_{n}$ Zerlegungsfunktionen des Gebietes $A_{1}$ sind, dass $w_{1}, \ldots, w_{n}$ und $w_{1}+\ldots+w_{n}$ Zerlegungsfunktionen des Gebietes $A_{2}$ sind, und dass bei $F$ der Funktion $z_{v}, v=1, \ldots, n$, die Funktion $w_{v}$ und der Funktion $z_{1}+\ldots+z_{n}$ die Funktion $w_{1}+\ldots+w_{n}$ zugeordnet wird. Es sei $U$ eine offene und zusammenhängende Teilmenge des Graphen $\mathscr{G}[F]$, derart dass $\Pi_{1}: U \rightarrow \Pi_{1}(U)$ biholomorph ist. Die Abbildung

$$
\Pi_{2} \circ \Pi_{1}^{-1}: \Pi_{1}(U) \rightarrow U \rightarrow A_{2}
$$

wird dann durch $n$ in $\Pi_{1}(U)$ holomorphe Funktionen

$$
w_{v}=\zeta_{v}\left(z_{1}, \ldots, z_{n}\right), \quad v=1, \ldots, n,
$$

bestimmt. Nach Satz 2 sind $\zeta_{v}$ und $z_{v}$ im engeren Sinne verwandt, das heisst, $\zeta_{v}$ ist nur von $z_{v}$ abhängig: $\zeta_{v}=\zeta_{v}\left(z_{v}\right), v=1, \ldots, n$. Weiter sind $\zeta_{1}+\ldots+\zeta_{n}$ und $z_{1}+\ldots+z_{n}$ im engeren Sinne verwandt, also

$$
\frac{d \zeta_{1}}{d z_{1}}=\ldots=\frac{d \zeta_{n}}{d z_{n}}
$$

Wegen $n \geqq 2$ folgt hieraus, dass

$$
\frac{d \zeta_{v}}{d z_{v}}=c, \quad v=1, \ldots, n
$$

wo $c$ eine Konstante ist, also

$$
\zeta_{\nu}=c z_{\nu}+b_{\nu} .
$$

Nach Proposition 3 gibt es irreduzible Polynome $P_{v}$ über $\mathcal{O}\left(A_{1}\right), \nu=$ $1, \ldots, n$, derart dass 


$$
P_{v}\left(w_{v} ; z_{1}, \ldots, z_{n}\right)=0 \quad \text { für } v=1, \ldots, n,
$$

wenn $\left(w_{1}, \ldots, w_{n}\right) \in F\left(z_{1}, \ldots, z_{n}\right)$. Es ist also

$$
P_{v}\left(c z_{v}+b_{v} ; z_{1}, \ldots, z_{n}\right)=0 \quad \text { für } v=1, \ldots, n
$$

in $\Pi_{1}(U)$ und damit im ganzen Gebiet $A_{1}$. Daraus folgt

$$
P_{v} \equiv \zeta_{\nu}-\left(c z_{v}+b_{v}\right)
$$

und $F$ ist also mit der Restriktion der durch

$$
\begin{gathered}
w_{1}=c z_{1}+b_{1}, \\
\vdots \\
w_{n}=c z_{n}+b_{n}
\end{gathered}
$$

gegebenen affinen Abbildung identisch. Damit ist der Satz bewiesen.

CoRollar. Jede holomorphe Überlagerungskorrespondenz $F: A_{1} \rightarrow_{k} A_{2}$ ist Vereinigung endlich vieler Restriktionen affiner Abbildungen.

Es seien jetzt $A \cong C^{n}$ ein euklidisches Polyedergebiet mit mindestens $n+1$ affinen Zerlegungen, von denen je $n$ unabhängig sind, und $\mathbb{S}$ die Gruppe seiner affinen Automorphismen. (ES ist offenbar eine endliche Gruppe.) Es gilt dann

Satz 4. Es seien $X$ ein komplexer Raum und $f: A \rightarrow X$ eine holomorphe Überlagerungsabbildung. Dann wirkt die Untergruppe

$$
\mathfrak{S}_{f}=\{\sigma \in \mathbb{S} \mid f \circ \sigma=f\}
$$

von (S) auf jeder Faser $f^{-1}$ (Punkt) transitiv und es gilt

$$
\operatorname{grad} f=\operatorname{ord} \mathfrak{G}_{f} \text {. }
$$

(Für die Definition von grad $f$ vgl. [5, S. 168]).

Jede holomorphe Utberlagerungsabbildung $g: A \rightarrow X$ ist von der Gestalt $g=f \circ \tau$ mit $\tau \in \mathbb{B S}$, und die Zuordnung $f \circ \tau \rightarrow \mathfrak{G}_{f} \tau$ definiert eine bijektive Korrespondenz zwischen holomorphen Überlagerungsabbildungen $A \rightarrow X$ und rechtseitigen Nebenklassen der Untergruppe $\mathfrak{H}_{f}$ von $\mathbb{E S}$.

BEwEIS. $f^{-1} \circ f: A \rightarrow_{k} A$ ist eine holomorphe Überlagerungskorrespondenz des Gebietes $A$ in sich. Für $\sigma \in \mathbb{B}$ gilt offenbar

$$
\sigma \subseteq f^{-1} \circ f \Leftrightarrow f \circ \sigma=f .
$$

Nach dem Corollar des Satzes 3 haben wir daher

$$
f^{-1} \circ f=\bigcup_{\sigma \in \mathfrak{q}_{f}} \sigma,
$$


woraus folgt, dass $\mathfrak{W}_{f}$ auf jeder Faser transitiv ist. Ein "allgemeiner" Punkt von $X$ hat vermöge $f$ offenbar ord $\mathfrak{G}_{f}$ Urbildpunkte, und es ist also $\operatorname{grad} f=\operatorname{ord} \mathfrak{G}_{f}$.

Es sei $g: A \rightarrow X$ eine holomorphe Überlagerungsabbildung. Dann ist $f^{-1} \circ g: A \rightarrow_{k} A$ eine holomorphe Überlagerungskorrespondenz von $A$. Es sei $\tau \in \mathbb{B S}$ mit $\tau \cong f^{-1} \circ g$. Dies bedeutet, dass $\tau(a)$ für jeden Punkt $\boldsymbol{a} \in A$ Originalpunkt des Punktes $g(\boldsymbol{a})$ vermöge $f$ ist, also dass $g=f \circ \tau$.

Endlich haben wir für $\tau_{1}, \tau_{2} \in \mathbb{S} \mathfrak{S}$

$$
\begin{aligned}
& f \circ \tau_{1}=f \circ \tau_{2} \Leftrightarrow f=f \circ \tau_{2} \circ \tau_{1}^{-1} \Leftrightarrow \tau_{2} \circ \tau_{1}^{-1} \in \mathscr{W}_{f} \\
& \Leftrightarrow \tau_{2} \in \mathfrak{H}_{f} \tau_{1} \quad \Leftrightarrow \mathfrak{H}_{f} \tau_{2}=\mathfrak{H}_{f} \tau_{1} .
\end{aligned}
$$

Damit ist der Satz bewiesen.

Man sieht noch, dass die Decktransformationsgruppe $\mathfrak{W}_{f_{\bullet} \tau}$ der Abbildung $f \circ \tau$ gleich $\tau^{-1} \mathfrak{G}_{f} \tau$ ist. Sie ist also in $\mathfrak{G S}$ zu $\mathfrak{W}_{f}$ konjugiert.

CoRollar: Wenn es holomorphe Überlagerungsabbildungen $A \rightarrow X$ gibt, so haben sie alle denselben Grad, und das Produkt ihrer Anzahl mit dem gemeinsamen Grad ist gleich der Anzahl der affinen Automorphismen von $A$.

Entsprechend gilt mit denselben Bezeichnungen:

Satz 5. Es seien $Y$ ein irreducibler komplexer Raum und $f: Y \rightarrow A$ eine holomorphe Überlagerungsabbildung. Dann ist jede holomorphe Überlagerungsabbildung $g: Y \rightarrow A$ von der Gestalt $g=\sigma \circ f$ mit $\sigma \in \mathbb{B}$.

Beweis. Es sei $\sigma \in \mathbb{S}$ mit $\sigma \subseteq g \circ f^{-1}$. Dann gibt es zu jedem Punkt $a \in A$ in der Menge $(\sigma \circ f)^{-1}(a)$ einen Punkt $y$, für welchen $g(y)=a$. Wir wählen eine offene einfach zusammenhängende Teilmenge $U$ des Gebietes $A$ derart, dass $f: f^{-1}(U) \rightarrow U$ lokaltopologisch ist. Die Menge $f^{-1}(U)$ ist dann die Vereinigung endlich vieler disjunkter offener Teilmengen $U_{j}$ des Raumes $Y$, von denen jede durch $f$ bijektiv auf $U$ abgebildet wird. Es folgt jetzt, dass $\sigma \circ f$ und $g$ in einer Menge $M \cong Y$, die dicht in einem offenen Teil von $Y$ liegt, übereinstimmen müssen. Also ist $g=\sigma \circ f$.

CoROLLAR. Wenn es holomorphe Überlagerungsabbildungen $Y \rightarrow A$ gibt, haben sie alle denselben Grad, und ihre Anzahl ist gleich die Anzahl der affinen Automorphismen von A.

\section{LITERATUR}

1. H. Grauert und R. Remmert, Komplexe Räume, Math. Ann. 136 (1958), 245-318.

2. H. Grauert, Ein Theorem der analytischen Garbentheorie und die Modulräume komplexer Strukturen, Inst. Hautes Etudes Sci. Publ. Math. 5 (1960). 
3. V. T. E. Osorio, Randeigenschaften eigentlicher holomorpher Abbildungen, Diss. Univ. München, 1961.

4. R. Remmert, Holomorphe und meromorphe Abbildungen komplexer Räume, Math. Ann. 133 (1957), 328-370.

5. R. Remmert und K. Stein, Eigentliche holomorphe Abbildungen, Math. Z. 73 (1960), 159-189.

6. H. Rischel, Ein Satz über eigentliche holomorphe Abbildungen von analytischen Polyedergebieten, Math. Scand. 14 (1964), 220-224.

7. W. Rothstein, Zur Theorie der analytischen Abbildungen im Raum zweier komplexer Veränderlichen, Diss. Univ. Münster, 1935.

8. K. Stein, Maximale holomorphe und meromorphe Abbildungen I, Amer. J. Math. 85 (1963), 298-315.

9. K. Stein, Maximale holomorphe und meromorphe Abbildungen II, erscheint in Amer. J. Math. 